\title{
Low lying hadron spectrum and chiral condensate with two flavours of naive Wilson fermions
}

\author{
Abhishek Chowdhury ${ }^{a}$, Asit K. De ${ }^{* a}$, Sangita De Sarkar ${ }^{a}$, A. Harindranath ${ }^{a}$, \\ Jyotirmoy Maiti ${ }^{b}$, Santanu Mondal $^{a}$, Anwesa Sarkar ${ }^{a}$ \\ ${ }^{a}$ Theory Division, Saha Institute of Nuclear Physics \\ 1/AF Bidhan Nagar, Kolkata 700064, India \\ ${ }^{b}$ Department of Physics, Barasat Government College, \\ 10 KNC Road, Barasat, Kolkata 700124, India \\ E-mail: asitk.de@saha.ac.in
}

\begin{abstract}
We study low lying hadronic spectrum with two degenerate flavours of naive Wilson fermions and unimproved Wilson gauge action using DDHMC algorithm as a function of PCAC quark mass at two different lattice spacings and different volumes. The scaling violations of the pionic observables and the nucleon mass are found to be negligible. The chiral behaviour of different observables are compared with NLO chiral perturbation theory predictions. Using the chiral Ward identity we calculate chiral condensate and it is found to be compatible with NLO chiral perturbation theory prediction.
\end{abstract}

The 30th International Symposium on Lattice Field Theory

June 24 - 29, 2012

Cairns, Australia

${ }^{*}$ Speaker. 


\section{Introduction}

Wilson fermions break all of the chiral symmetry explicitly in the kinetic part of the lattice action and naive (unimproved) Wilson fermions have $\mathscr{O}(a)$ cut-off effects. As a result, fermion masses have additive mass renormalization in addition to the usual multiplicative renormalization. Absence of any chiral symmetry also means possible presence of arbitrarily small eigenvalues of the Wilson-Dirac operator (the so-called problem of exceptional configurations) resulting in great difficulty in inversion of the fermion matrix and in turn the updation of the gauge field configurations.

Previous investigations of lattice QCD (LQCD) with naive Wilson fermions showed significant presence of cut-off effects in hadronic observables. As a result, emergence of the chiral regime of QCD and consistency of the low lying spectrum with chiral perturbation theory from LQCD with naive Wilson quarks have remained a big question mark. In addition, suppression of the topological susceptibility with decreasing quark mass also could not be demonstrated. There has been persistent doubt about Wilson LQCD being able to produce the correct chiral limit (see [1]).

The previous numerical investigations of LQCD with naive Wilson quarks, however, involved serious compromises in the form of one or more of the following: quenched approximation, large lattice spacings, large quark masses, small volumes. With the standard HMC algorithm and the existing computing power of the time, it was not possible to conquer the problem of exceptional configurations and achieve dynamical simulations with naive Wilson quarks at relatively large volumes and smaller lattice spacings and smaller quark masses.

With the development of improved versions of the standard HMC algorithm like the domain decomposed HMC (DD-HMC) [2], it is now affirmed that Wilson fermions are safe from exceptional configurations if the volume is relatively large. This, along with significant advancement of computing power, has made it possible for Wilson LQCD to be simulated at large volumes, small lattice spacings and small quark masses and has, in a way, revived interest in LQCD with Wilson fermions.

In this work, we are going to present preparatory results of our on-going investigation of the chiral regime of LQCD with two flavors of naive Wilson fermions and (unimproved) Wilson gauge action. The simulations are done so far at two lattice spacings in the region of $0.05-0.07 \mathrm{fm}$ where many of the modern simulations of LQCD with improved actions are carried out. The interesting question is of course whether at these lattice spacings naive Wilson LQCD shows large scaling violations in the low lying hadronic spectrum, and whether a chiral regime emerges at moderate quark masses as used so far in this work. As our preparatory and qualitative results will show, the outcome is promising.

From essentially the same set of data, we have recently shown $[3,4]$ the suppression of the topological susceptibility with decreasing quark masses and also with decreasing volumes, as a probe of emergence of chiral properties of naive Wilson fermions. To understand the mechanisms working underneath these suppressions, we have also studied the 2-point topological charge density correlator in somewhat detail [5]. A study of the autocorrelations of a variety of hadronic and gauge operators has also been carried out [6]. 


\section{Details of Simulation and Observables}

Gauge configurations are generated at two gauge couplings by means of DD-HMC algorithm using unimproved Wilson fermion and gauge actions with $n_{f}=2$ mass degenerate quark flavors. At $\beta=5.6$ the lattice volumes are $24^{3} \times 48$ and $32^{3} \times 64$ and at $\beta=5.8$ the only lattice volume so far is $32^{3} \times 64$. The number of thermalized configurations at the moment ranges approximately from 5000 to 14000 (the number has significantly increased for some of our data points since the time of the presentation at LATTICE 2012 with introduction of a few new points). The lattice parameters and simulation statistics are given in Table 1. The average acceptance rates at the accept/reject stage of the DD-HMC ranges between $75-98 \%$. For our recent runs, hadronic measurements are carried out once in every 6 trajectories for the $\beta=5.6$ runs at $24^{3} \times 48$, and once in 8 trajectories for the $\beta=5.8$ runs. These gaps in measurements are supported very well by our study of the respective autocorrelations of the hadronic observables. For the older runs (runs with $N_{2}=8$ in Table 1), hadronic measurements were done once in every 24 and 32 trajectories respectively at $24^{3} \times 48$ and $32^{3} \times 64$.

For pion and nucleon we compute the following zero spatial momentum 2-point correlators in Euclidean time $t$ functions

$$
C(t)=\left\langle 0\left|\mathscr{O}_{1}(t) \mathscr{O}_{2}(0)\right| 0\right\rangle
$$

where, for the nucleon correlator, $\mathscr{O}_{1} \mathscr{O}_{2} \equiv \mathrm{N} \overline{\mathrm{N}}$ with $\mathrm{N}=\left(q_{d}^{T} C \gamma_{5} q_{u}\right) q_{u}$, and for the pion correlators, $\mathscr{O}_{1} \mathscr{O}_{2} \equiv P P^{\dagger}, A A^{\dagger}, A P^{\dagger}$ or $P A^{\dagger}$ with $P=\bar{q}_{i} \gamma_{5} q_{j}$ (pseudoscalar density) and $A$ corresponds to $A_{4}=$ $\bar{q}_{i} \gamma_{4} \gamma_{5} q_{j}$ (fourth component of the axial vector current). Here $i$ and $j$ are flavor indices for the light quarks and for the charged pion $i \neq j$. For pion we use point source and point sink and for nucleon we use wall source and point sink. Unless otherwise stated, 20 HYP smearing steps with optimized smearing coefficients $\alpha_{1}=0.75, \alpha_{2}=0.6$ and $\alpha_{3}=0.3$ [8] are used for the gauge observables. The pion decay constant $F_{\pi}$ and the quark mass $m_{q}$ are defined from PCAC or the axial Ward identity (AWI).

We have also measured the rho and the delta propagators. As already mentioned, new gauge configurations have been generated and new measurements have been performed over the increased set of data since the time of the LATTICE 2012 symposium, and the analysis is still not completed for a significant part of the observables. Hence, this write-up will have to contain some old data and some new data in part. We shall not include the rho and the delta data in this write-up since the new analysis is yet to be completed.

In this write-up we present results for all points in Table 1 except for the runs $C_{3}$ and $D_{5 b}$. A full account and analysis of the current data is available in [7]. The lowest pion mass presented here is about $290 \mathrm{MeV}$. There are other low statistics runs with lower pion masses (not included in Table 1), and they will not be presented here.

\section{Results}

\subsection{Cut-off Effects}

As will be seen in the next subsection, the lattice spacings obtained are $a \sim 0.072 \mathrm{fm}$ and 0.057 $\mathrm{fm}$ respectively at $\beta=5.6$ and 5.8. Both lattices are relatively fine; however, they also differ in 


\begin{tabular}{|l|c|l|l|l|l|l|l|}
\multicolumn{8}{c|}{$\beta=5.6$} \\
\hline tag & lattice & $\kappa$ & block & $N_{2}$ & $N_{\text {cfg }}$ & $\tau$ & $r_{0} \times m_{\pi}$ \\
\hline$B_{1 b}$ & $24^{3} \times 48$ & 0.1575 & $12^{2} \times 6^{2}$ & 18 & 13128 & 0.5 & $1.7719(38)$ \\
$B_{3 b}$ &,, & 0.158 & $12^{2} \times 6^{2}$ & 18 & 13646 & 0.5 & $1.2542(58)$ \\
$B_{4 b}$ &, & 0.158125 & $12^{2} \times 6^{2}$ & 18 & 11328 & 0.5 & $1.0925(58)$ \\
\hline$C_{1}$ & $32^{3} \times 64$ & 0.15775 & $8^{3} \times 16$ & 8 & 6844 & 0.5 & $1.5345(54)$ \\
$C_{2}$ &,, & 0.158 & $8^{3} \times 16$ & 8 & 7576 & 0.5 & $1.2590(59)$ \\
$C_{3}$ &, & 0.158125 & $8^{3} \times 16$ & 8 & 8768 & 0.5 & $1.1010(60)$ \\
$C_{4}$ &, & 0.15815 & $8^{3} \times 16$ & 8 & 9556 & 0.5 & $1.0697(57)$ \\
$C_{5}$ &, & 0.15825 & $8^{3} \times 16$ & 8 & 11520 & 0.5 & $0.9343(55)$ \\
$C_{6}$ &,, & 0.1583 & $8^{3} \times 16$ & 8 & 4384 & 0.25 & $0.8476(99)$ \\
\hline \multicolumn{7}{|c|}{$\beta=5.8$} \\
\hline$t a g$ & lattice & $\kappa$ & block & $N_{2}$ & $N_{c f g}$ & $\tau$ & $r_{0} \times m_{\pi}$ \\
\hline$D_{1 a}$ & $32^{3} \times 64$ & 0.1543 & $8^{3} \times 16$ & 8 & 9600 & 0.5 & $1.3259(76)$ \\
$D_{2 b}$ &,, & 0.15445 & $8^{3} \times 16$ & 24 & 4800 & 0.5 & $1.1138(73)$ \\
$D_{3 a}$ &, & 0.15455 & $8^{3} \times 16$ & 8 & 12160 & 0.5 & $0.9968(87)$ \\
$D_{4 b}$ &,, & 0.15462 & $8^{3} \times 16$ & 24 & 7528 & 0.5 & $0.8637(81)$ \\
$D_{5 b}$ &, & 0.15466 & $8^{3} \times 16$ & 24 & 3760 & 0.5 & $0.8360(131)$ \\
$D_{6 b}$ &,, & 0.1547 & $8^{3} \times 16$ & 24 & 4256 & 0.5 & $0.6851(181)$ \\
\hline \hline
\end{tabular}

Table 1: Lattice parameters, simulation statistics and pion mass $\left(m_{\pi}\right)$ in unit of Sommer parameter $\left(r_{0}\right)$. Here block, $N_{2}, N_{c f g}, \tau$ refers to DD-HMC block, step number for the force $F_{2}$, number of DD-HMC configurations and the Molecular Dynamics trajectory length respectively.

value significantly. A major concern with naive Wilson quarks obviously is regarding the amount of visible cut-off effects in the hadronic observables at these lattice spacings. Fig. 1 presents nucleon mass and squared pion mass in units of the Sommer parameter $r_{0}$ as the renormalized quark mass is varied at both values of the gauge coupling $(\beta=5.6,5.8)$. There are no significant cut-off effects seen in these figures showing combined hadron mass data at two different lattice spacings.

\subsection{The Lattice Spacing}

We estimate the Sommer parameter $r_{0}$ by plotting the ratio of the nucleon mass to the pion mass in dependence of the pion mass in the units of the Sommer parameter and use the physical values of the masses to determine $r_{0}$. Our current best estimate of the Sommer parameter is, $r_{0} \sim 0.46 \mathrm{fm}$. Obviously, there are uncertainties in this estimate. The biggest source of these uncertainty is the chiral extrapolation using a phenomenological chiral fit (dropping a few terms from the NLO baryon chiral perturbation theory). The fit (shown in Fig. 2 (left) for $\beta=5.6$ ) is decent; however, a few more data deeper into the chiral region would be very welcome for a more faithful estimation.

The lattice spacings are then estimated by using the values in the chiral limit of the ratios $a / r_{0}$ (obtained from the rotationally corrected static potential) for each $\beta$. The plots versus the lattice quark masses in Fig. 2 (right) are nicely linear for small enough quark masses with smalish slopes. 

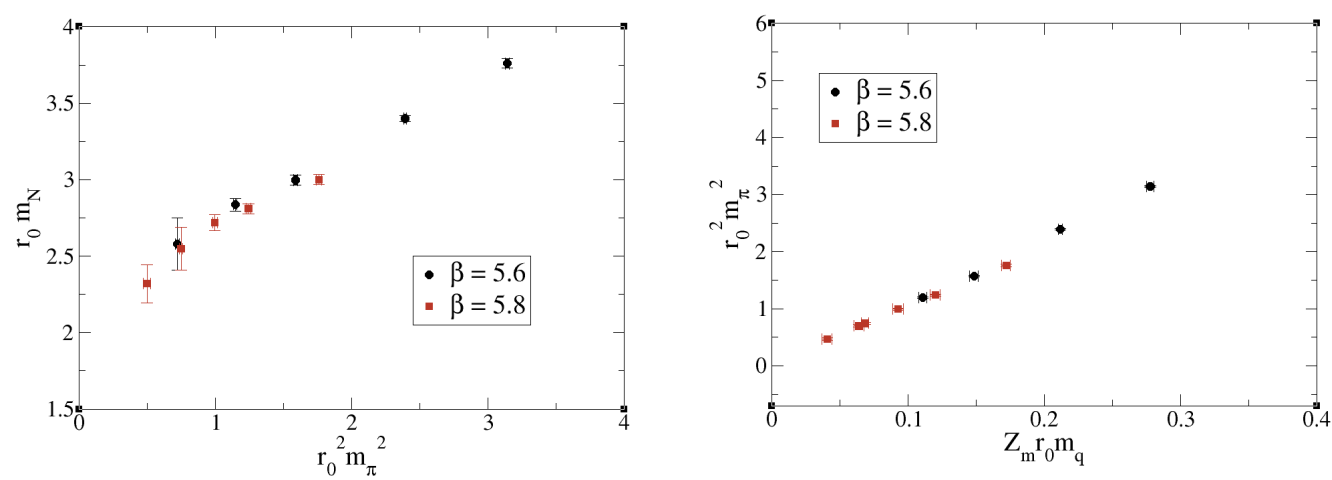

Figure 1: Combined data of nucleon mass and squared pion mass at $\beta=5.6(a=0.072 \mathrm{fm})$ and at $\beta=5.8$ $(a=0.057 \mathrm{fm})$.
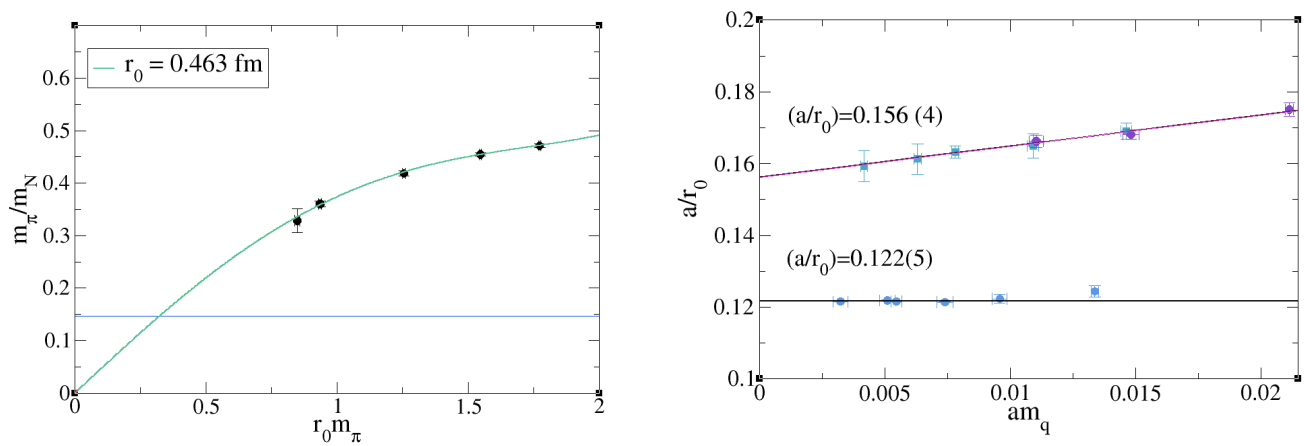

Figure 2: Estimating the lattice spacing at both gauge couplings. The left figure is at $\beta=5.6$ while the right figure shows data at both gauge couplings with the top one describing data at the lower $\beta$

Particularly, for $\beta=5.8$ the slope is nearly zero, showing practically no dependence of $a / r_{0}$ on the lattice quark mass $a m_{q}$.

\subsection{Chiral Extrapolation}

Chiral extrapolation of the squared pion mass in units of the Sommer parameter is shown in Fig. 3 (left) using NLO chiral perturbation theory in the continuum with $F_{\pi}=86 \mathrm{MeV}$ (value in the chiral limit) taken as input. We have decided to show both plots in Fig. 3 in a different way than during the LATTICE2012 presentation where for $\beta=5.6$ infinite volume extrapolations were carried out. That finite volume analysis, however, has helped us identify the data at $24^{3} \times 48$ which are suitable for chiral extrapolations. In the current plots, data from $\beta=5.8$ (only $32^{3} \times 64$ available) and suitable $24^{3} \times 48$ data from $\beta=5.6$ have been included, keeping in mind that the physical volumes for both the gauge couplings are nearly the same (approximately $\left.(1.8 \mathrm{fm})^{3}\right)$. The same strategy was used also in the previous figures. The NLO chiral fit was done dropping the points with the two heaviest pion masses (corresponding to $\kappa=0.1575$ and 0.15775 at $\beta=5.6$ ). Fig. 3 (right) which plots the ratio of the squared pion mass to the quark mass takes the value of $r_{0} B$ from the fit of the left plot as input. The values of the low energy constants from these chiral 

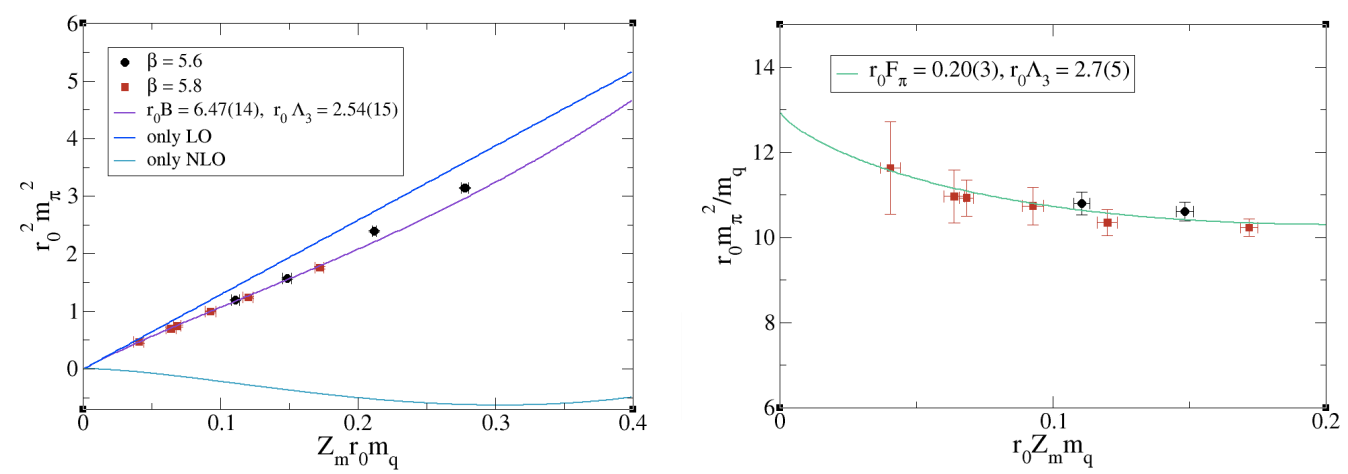

Figure 3: NLO chiral extrapolation of the squared pion mass and the ratio of the squared pion mass to the quark mass

fits are quite reasonable. The chiral condensate extracted from the fits, $\Sigma^{1 / 3} \sim 270 \mathrm{MeV}$, is very much consistent with findings by other LQCD groups using various forms of improved actions.

\subsection{Chiral Condensate from the Ward Identity}

Because of explicit breaking of chiral symmetry in the kinetic part of the action, a direct computation of the chiral condensate in Wilson LQCD will not produce the correct value in the chiral limit. However, one can use the chiral Ward Identity using the operator $\mathscr{O}=P^{b}=\bar{\psi} \lambda^{b} \gamma_{5} \psi$ to obtain

$$
\langle\bar{\psi} \psi\rangle \delta^{a b}=2 m_{q} \int d^{4} x\left\langle P^{a}(x) P^{b}(0)\right\rangle
$$

from which one gets

$$
\langle\bar{\psi} \psi\rangle=2 m_{q} \frac{C_{P P}}{m_{\pi}}=C_{A P}
$$

where $C_{P P}$ and $C_{A P}$ are the coefficients of the $\mathrm{PP}$ and the $\mathrm{AP}$ pion correlators respectively.

Fig. 4 plots the quark condensate in dependence of the renormalized quark mass in lattice units. This plot uses the data available at the time of the LATTICE 2012 symposium and hence has less statistics in general. The new data with more statistics has not yet been plotted for the condensate. The value in the chiral limit from a reasonable NLO chiral fit is, $\Sigma^{1 / 3} \sim 230 \mathrm{MeV}$ (renormalized).

The renormalization constants used in this work has been taken from [9].

In conclusion, Wilson LQCD seems to be devoid of significant scaling violations at the lattice spacings investigated in the current work and produce qualitatively the desired chiral properties of QCD.

\section{Acknowledgements}

Numerical calculations are carried out on Cray XD1 and Cray XT5 systems supported by the 10th and 11th Five Year Plan Projects of the Theory Division, SINP under the DAE, Govt. of 


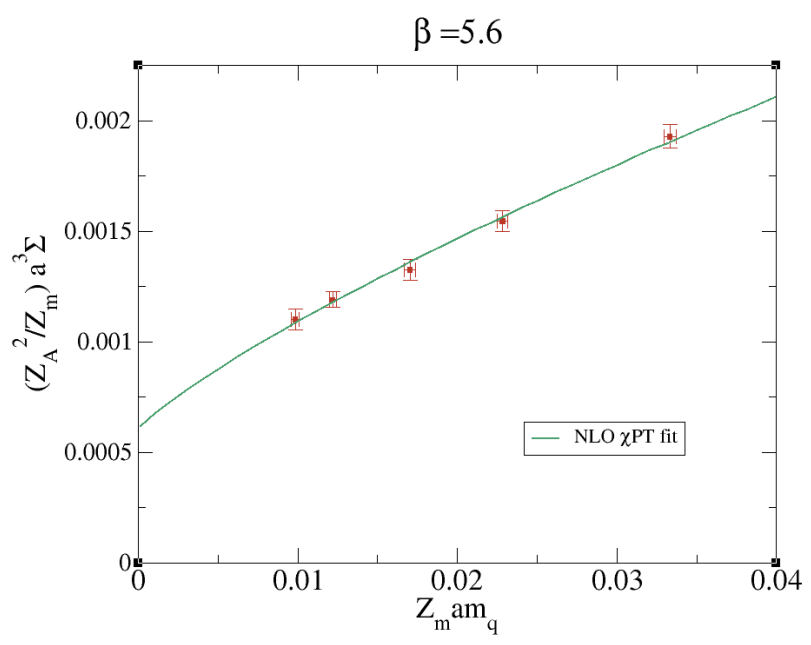

Figure 4: Condensate versus quark mass in lattice units at $\beta=5.6$.

India. We thank Richard Chang for the prompt maintenance of the systems and the help in data management. This work was in part based on the public lattice gauge theory codes of the MILC collaboration [10] and Martin Lüscher [2].

\section{References}

[1] Contribution by Kim Splittorff in the PoS LATTICE 2012.

[2] M. Lüscher, Comput. Phys. Commun. 156, 209-220 (2004). [hep-lat/0310048]; M. Lüscher, Comput. Phys. Commun. 165, 199-220 (2005). [hep-lat/0409106].

http://luscher.web.cern.ch/luscher/DD-HMC/index.html

[3] A. Chowdhury, A. K. De, S. De Sarkar, A. Harindranath, S. Mondal, A. Sarkar and J. Maiti, Phys. Lett. B 707, 228 (2012) [arXiv:1110.6013 [hep-lat]].

[4] A. Chowdhury, A. K. De, S. De Sarkar, A. Harindranath, S. Mondal, A. Sarkar and J. Maiti, PoS LATTICE 2011, 099 (2011) [arXiv:1111.1812 [hep-lat]].

[5] A. Chowdhury, A. K. De, A. Harindranath, J. Maiti and S. Mondal, "Topological charge density correlator in Lattice QCD with two flavours of unimproved Wilson fermions", arXiv:1208.4235v1 [hep-lat], accepted for publication in JHEP; see also contribution by Santanu Mondal in PoS LATTICE 2012.

[6] A. Chowdhury, A. K. De, S. De Sarkar, A. Harindranath, J. Maiti, S. Mondal and A. Sarkar, arXiv:1209.3915 [hep-lat]; see also contribution by Santanu Mondal in PoS LATTICE 2012.

[7] A. Chowdhury, A. K. De, S. De Sarkar, A. Harindranath, J. Maiti, S. Mondal and A. Sarkar, arXiv:1212.0717 [hep-lat].

[8] A. Hasenfratz, F. Knechtli, Phys. Rev. D64, 034504 (2001). [hep-lat/0103029].

[9] D. Becirevic, B. Blossier. Ph. Boucaud, V. Gimenez, V. Lubicz, F. Mescia, S. Simula, C. Tarantino, Nucl. Phys. B 734 (2006) 138.

[10] http://physics.indiana.edu/ sg/milc.html 